\title{
Establishment and validation of prognostic nomograms to predict overall survival and cancer-specific survival for patients with osteosarcoma
}

\author{
Qian-Kun YANG ${ }^{1, *}$, Qing-Yuan LAI ${ }^{2, *}$, Yi WANG ${ }^{3, *}$, Yang WANG ${ }^{4, *}$, Zhong-Xiang YAO ${ }^{5}$, Xiao-Jing ZHANG ${ }^{1, *}$ \\ ${ }^{1}$ Department of Bone and Soft Tissue Surgery, Cancer Hospital of China Medical University, Liaoning Cancer Hospital and Institute, Shenyang, \\ China; ${ }^{2}$ Department of Radiology, Cancer Hospital of China Medical University, Liaoning Cancer Hospital and Institute, Shenyang, China; \\ ${ }^{3}$ The First School of Clinical Medicine, MuDanJiang Medical University, MuDanJiang, China; ${ }^{4}$ Laboratory of Aging Research and Cancer Drug \\ Target, State Key Laboratory of Biotherapy, National Clinical Research Center for Geriatrics, West China Hospital, Sichuan University, Cheng- \\ du, China; ${ }^{5}$ Department of Physiology, Army Medical University, Chongqing, China
}

${ }^{*}$ Correspondence: zhangxiaojing@cancerhosp-ln-cmu.com

${ }^{*}$ Contributed equally to this work.

Received June 17, 2020 / Accepted October 9, 2020

\begin{abstract}
This study aimed to develop and validate nomograms predicting the survival of osteosarcoma patients from the SEER database and our hospital. Data of 1,066 osteosarcoma patients from the SEER database were randomly divided into a development cohort $(n=800)$ and validation cohort one $(n=266)$. Another cohort of 126 patients from our hospital was utilized as validation cohort two. Univariate and multivariate Cox analyses were performed to identify the independent prognostic factors for overall survival (OS) and cancer-specific survival (CSS). Nomograms predicting the 3- and 5-year OS and CSS probability were constructed and validated. The predictive performances of the established nomograms were evaluated by the concordance index (C-index) and the calibration plot. Variables of age, surgical stage, surgery, grade, tumor site, and tumor size were identified as independent prognosticators for OS and CSS in Cox analyses. The C-indexes for OS and CSS in the development cohort were 0.818 and 0.829 . Comparatively, the C-indexes for OS and CSS were 0.843 and 0.834 , 0.736 and 0.782 for validation cohort one and two, respectively. Calibration plots showed excellent consistency between nomogram prediction and actual survival. Nomograms based on the SEER database are of high accuracy and can serve as a reliable tool for individualized consultation and survival prediction in osteosarcoma patients.
\end{abstract}

Key words: nomogram, osteosarcoma, overall survival, cancer-specific survival

Osteosarcoma is the most common primary malignancy of bones which accounts for $35 \%$ of all primary malignant bone tumors, and it typically arises in children and adolescents younger than 24 years of age, with an estimated incidence of $0.34 / 100,000$ per year $[1,2]$. Prior to the introduction of chemotherapy, amputation was the main therapeutic measure for osteosarcoma patients but with low quality of life and unfavorable survival rates. In addition, 80 to 90 percent of osteosarcoma patients developed metastatic disease despite the achievement of local tumor control and finally died of metastases. It was surmised and subsequently demonstrated that the vast majority of osteosarcoma patients had developed the subclinical metastatic disease at the time of diagnosis, despite the absence of overt clinical symptoms [3]. Currently, neoadjuvant chemotherapy followed by surgical resection of the primary lesions has been established and demonstrated as the standard therapeutic strategy for newly diagnosed non-metastatic osteosarcoma [4]. With multi-disciplinary treatments, the 5-year survival rate for these patients has risen from less than $20 \%$ to approximately $70 \%$ [5]. Nevertheless, available and effective options are still insufficient and the outcomes are still poor for metastatic and recurrent osteosarcoma patients [6,7]. Better comprehension and identification of prognostic factors of osteosarcoma can provide us more information to select therapeutic interventions, which will contribute to improvement in the quality of life and prolonging survival in patients with osteosarcoma.

A series of factors have been reported in the literature to have predictive or prognostic values for the survival of patients with osteosarcoma. These predictive factors can be roughly divided into seven groups based on their characteristics, including 1) inflammation-related predictive variables, such as serum C-reactive protein (CRP), pre-treatment neutrophil-to-lymphocyte ratio (Pre-NLR), absolute lymphocyte count (ALC), Glasgow prognostic score (GPS), platelet-lymphocyte ratio (PLR), lymphocyte-monocyte ratio 
(LMR), and neutrophil-platelet score (NPS) [8-10]; 2) nutrition-related predictive factors or scores, such as body mass index (BMI), prognostic nutritional index (PNI), Geriatric Nutritional Risk Index (GNRI) [11-13]; 3) aberrant expression of specific proteins, such as cell-cycle kinase inhibitor p27, cyclin-dependent protein kinase 9 (CDK9), motilityrelated protein-1 (MRP-1)/CD9 [14-16]; 4) upregulation or downregulation of microRNAs, non-coding RNA, and circular RNAs, such as serum microRNA-375, microRNA17, long non-coding RNA HAGLROS, circulating hsa circ_0081001 [17-21]; 5) abnormalities of constituents in the tumor microenvironment, such as tumor-infiltrating macrophages, CD8-positive cytotoxic lymphocytes, immune infiltrations, PD-L1 expression, dysregulation of M1/M2 macrophages ratio [22-25]; 6) features of the disease, such as TNM stage, AJCC stage, lymph node involvement, histopathologic features, metastasis, age at diagnosis, tumor grade, tumor site, surgical margins, pathological fractures, etc. [26-32]; 7) predictors from imaging examination, such as maximum and peak standardized uptake value (SUVmax, SUVpeak) on 18F-fluoro-2-deoxyglucose positron emission tomography/ computed tomography (18FDG-PET/CT), kinetic parameters of dynamic contrast-enhanced magnetic resonance imaging (DCE-MRI), including the influx volume transfer constant $[\mathrm{K}(\operatorname{trans})]$, the relative extravascular extracellular space $[v(e)]$, the relative vascular plasma space $[v(p)]$ and the efflux rate constant [k(ep)] [33-35]. As reported in previous studies, these variables are significantly associated with the survival of osteosarcoma patients in statistical analyses. However, the survival of an osteosarcoma patient is determined by multiple factors, and therefore single predictorbased survival prediction is usually inaccurate and unreliable. On account of this fact, it is reasonable to construct a new evaluation system, which can integrate these pivotal prognostic factors together.

Nomogram is a newly developed prediction tool that has been widely used to estimate the survival probability in a variety of tumors, including lung cancer, breast cancer, and gastric cancer [36-38]. A predictive nomogram is an ocular, reliable, and effective tool based on multivariate regression models, which can provide intuitive and graphic calculating scales to estimate the survival probability of patients with osteosarcoma. The outstanding characteristics of nomogram in incorporating these key prognostic factors together significantly improve the accuracy and reliability in estimating medium- and long-term survival, as well as risk stratification of osteosarcoma patients.

The Surveillance, Epidemiology, and End Results (SEER) dataset is an open, updated database, which has collected anonymized clinical information of multiple malignancies from 1983 to now [39]. SEER database has covered approximately $30 \%$ of the overall US population and is composed of eighteen cancer registries [40]. In this study, we first established effective prognostic nomograms to estimate the 3 - and 5 -year overall survival (OS) and cancer-specific survival
(CSS) rates for patients with osteosarcoma based on the data from the SEER database. Then, we validated the developed nomograms internally and externally with data from the SEER database and our hospital.

\section{Patients and methods}

Data source. Two datasets, which respectively came from the SEER database (patients diagnosed with osteosarcoma from 2010 to 2015) and Cancer Hospital of China Medical University (patients diagnosed with osteosarcoma between 2010 to 2016) were utilized for this study. The SEER ${ }^{\star}$ Stat software (version 8.3.6; NCI, Bethesda, MD, USA) was used to extract the information of each osteosarcoma patient from the SEER database. All the data are anonymous, and therefore the requirement for informed consent was waived.

Inclusive and exclusive criteria for osteosarcoma patients. The inclusion criteria were as follows: 1) diagnosed with osteosarcoma as primary malignancy (the International Classification of Disease for Oncology [ICD-O] were 9180-9187, 9192-9194 and 9200); 2) diagnosed between the year of 2010 to $2015 ; 3)$ the primary lesion sites were limited to extremities (long or short bones of the lower or upper extremities) and axial bones (including skull, spine, ribs, sternum, or pelvis); 4) confirmation of histologic subtypes of osteosarcoma; 5) with detailed information for race, surgical stage, surgery, histologic grade, AJCC stage; 6) with known survival months, survival status, and cause of death.

The exclusion criteria included: 1) no information for race, surgery, and TNM stage; 2) unknown or unspecific AJCC stage; 3 ) no detailed histologic grade; 4) unknown tumor size or surgical stage. The study design and process of collecting patients were shown in a flow diagram (Figure 1).

Variables included in this study. The potential prognostic variables for this study mainly included age, gender, race, tumor site, surgical stage, surgery, histologic grade, histologic subtypes, AJCC stage, tumor size, survival months, survival status, and cause of death. The X-tile software (Yale University, New Haven, CT, USA) was used to identify the optimal cut-off values to categorized continuous variables of age and tumor size.

Statistical analysis. X-tile software was used to categorize continuous variables of age and tumor size. Categorical variables were presented as frequencies and percentages. Chi-square test or Fisher exact test was used to compare the differences of variables of cohorts. The primary endpoints for the current study were overall survival (OS) and cancerspecific survival (CSS). OS was defined as the time from diagnosis to death from any cause, and CSS was calculated from the time of diagnosis to death attributed to osteosarcoma only. A two-sided p-value $<0.05$ was considered as statistically significant.

Identification of prognosticators for survival. Univariate and multivariate Cox proportional hazards regression analyses were used to identify independent prognostic factors 


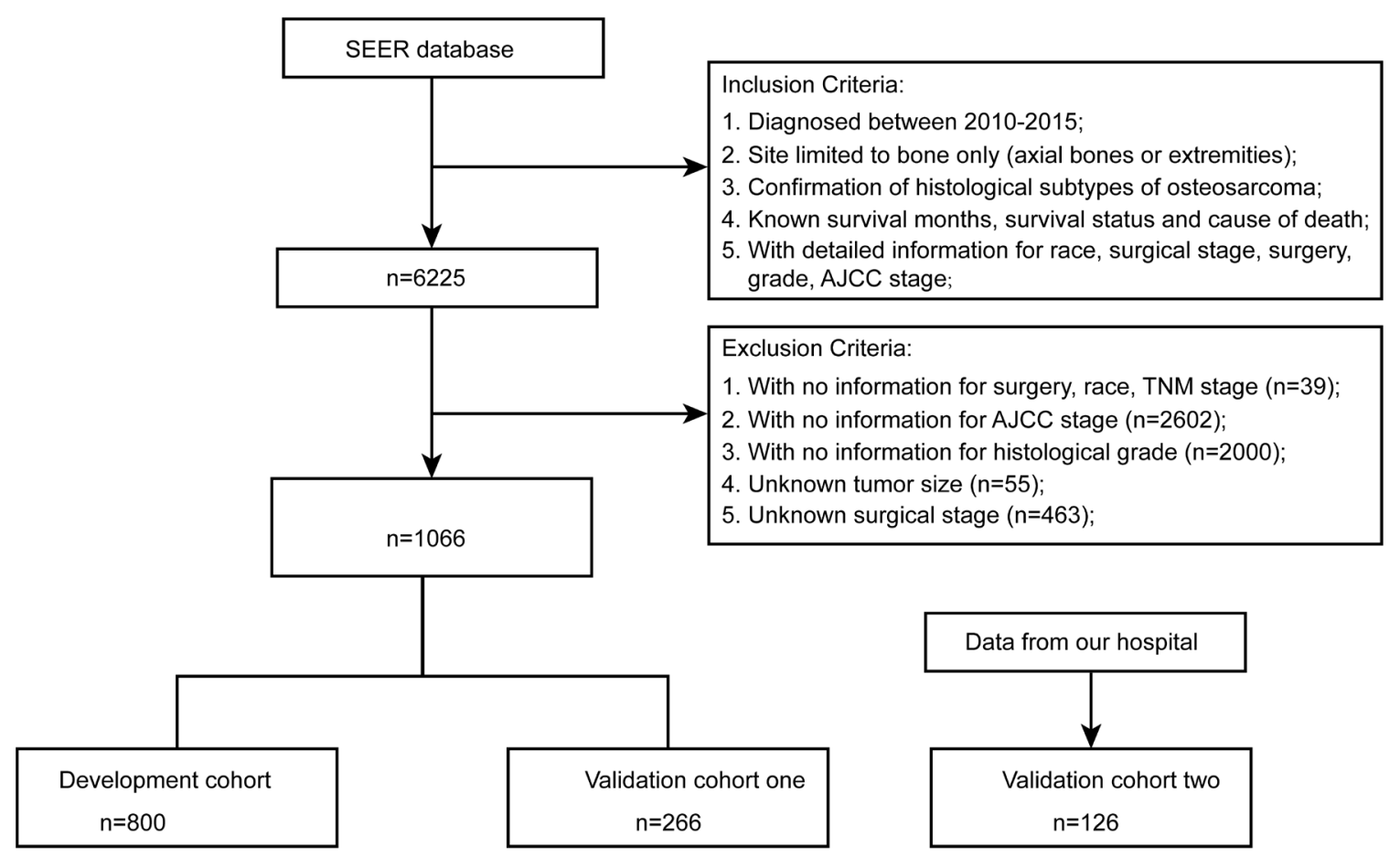

Figure 1. The flow chart of the inclusion and exclusion criteria of this study.

for OS and CSS from the potential prognostic factors. The hazard ratio and its corresponding $95 \%$ confidence interval of each variable were also calculated and presented.

Establishment and validation of predictive models for OS and CSS. The prognostic nomograms for 3- and 5-year OS and CSS were established based on the prognosticators determined in univariate and multivariate Cox analyses. Internal validation (data from the development cohort) and external validation (data from validation cohort one and two) were performed with 500 bootstrap resamples to prevent overfitting and to get a relatively unbiased estimation. Harrell's concordance-index (C-index) was utilized to evaluate the performance of these established predictive nomograms. Calibration curves were also constructed to assess the consistency between predicted and actual survival internally and externally.

Software for statistical analysis. Several pivotal softwares were utilized in our study, including $\mathrm{SEER}^{*}$ Stat software (Version 8.3.6; NCI, Bethesda, USA), IBM SPSS Statistics 24 (IBM Corp, Armonk, NY, USA), and R software (version 3.6.0). SEER ${ }^{\star}$ Stat software was used to extract the information of each osteosarcoma patient from the SEER database. Chi-squared tests, Fisher exact tests, univariate and multivariate Cox analyses were conducted by SPSS. R software was used to establish nomograms and calibration plots with the help of some essential packages, such as rms, foreign, survival, etc.

\section{Results}

Baseline characteristics of osteosarcoma patients. A total of 6,225 osteosarcoma patients were preliminarily collected from the SEER database between 2010 to 2015 . Based on the inclusion and exclusion criteria, an entire cohort of 1,066 osteosarcoma patients was finally enrolled for this study, and they were further divided into the development cohort and the validation cohort one. Another cohort of 126 osteosarcoma patients from our hospital was used as the validation cohort two (Figure 1).

The optimal cut-off values for age and tumor size identified by X-tile software were 26 and 61 years, and 9.6 and 20.5 $\mathrm{cm}$, respectively (Figures 2A-2D). The details of the clinicopathological features of all patients included in this study are presented in Table 1. No significant differences in clinicopathological features were observed between the development cohort and the two validation cohorts.

Identification of prognostic factors for OS and CSS. The dataset from the development cohort was used to determine the independent prognostic factors for OS and CSS. In univariate analyses, variables of age, gender, tumor site, surgical stage, surgery, grade, histology, AJCC stage, node involvement, metastasis, tumor size were all significantly associated with OS (all $\mathrm{p}<0.05)$. These factors were then selected to perform multivariate Cox analysis. In multivariate Cox analysis, variables of age, surgical stage, surgery, grade, 

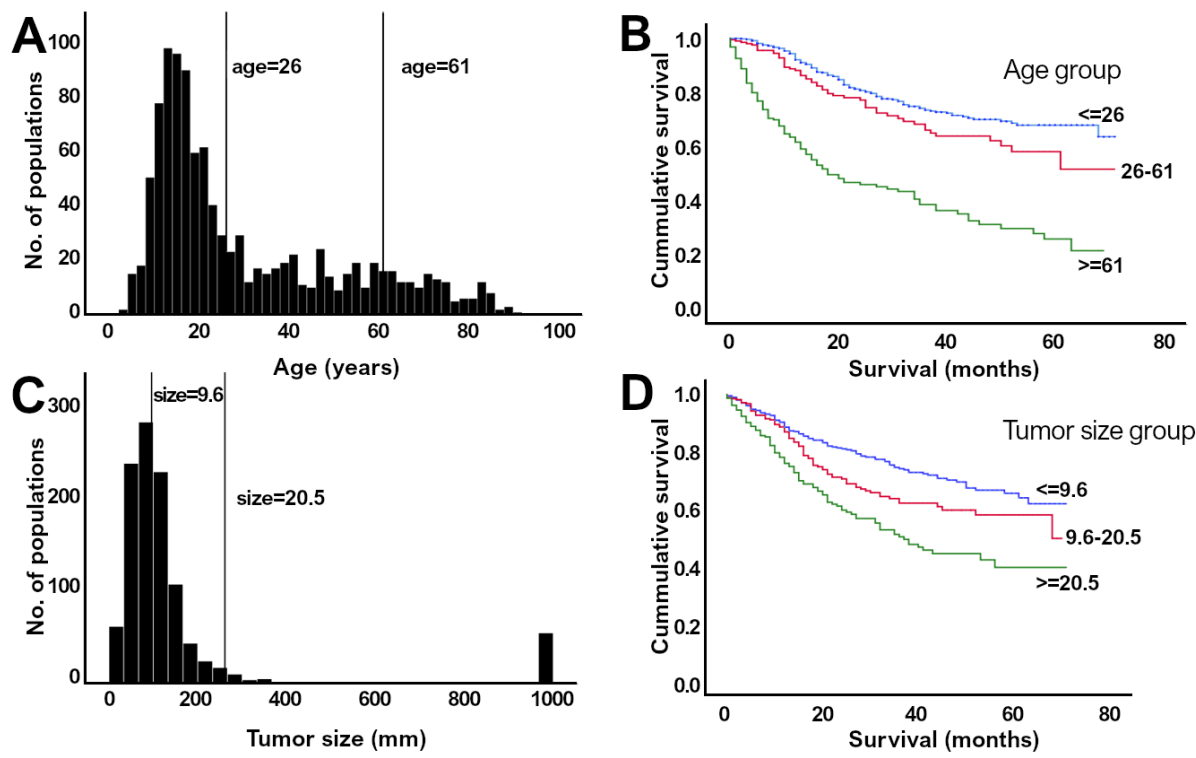

Figure 2. The optimal cut-off values of age at diagnosis (A), and tumor size (C) identified by the X-tile software. The survival curve analysis for age (B), and tumor size (D) based on the optimal cut-off values groupings.

Table 1. Baseline characteristics of all patients in this study.

\begin{tabular}{|c|c|c|c|c|c|}
\hline Variables & $\begin{array}{l}\text { Total cohort } \\
\mathrm{n}=1192 \\
\text { No. }(\%)\end{array}$ & $\begin{array}{c}\text { Development cohort } \\
\text { n }=\mathbf{8 0 0} \\
\text { No. }(\%)\end{array}$ & 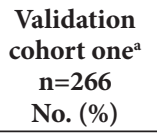 & $\begin{array}{c}\text { Validation } \\
\text { cohort two } \\
\text { n=126 } \\
\text { No. }(\%) \\
\end{array}$ & p-value \\
\hline Age & & & & & 0.500 \\
\hline$\leq 26$ & $716(60.1 \%)$ & $478(59.8 \%)$ & $166(62.4 \%)$ & $72(57.1 \%)$ & \\
\hline $26-61$ & $316(26.5 \%)$ & $220(27.5 \%)$ & $60(22.6 \%)$ & $36(28.6 \%)$ & \\
\hline$\geq 61$ & $160(13.4 \%)$ & $102(12.7 \%)$ & $40(15.0 \%)$ & $18(14.3 \%)$ & \\
\hline Median (range) & $16(3-94)$ & $17(3-93)$ & $17(3-94)$ & $18(4-78)$ & \\
\hline Gender & & & & & 0.259 \\
\hline Male & $656(55.0 \%)$ & $433(54.1 \%)$ & $145(54.4 \%)$ & $78(61.9 \%)$ & \\
\hline Female & $536(45.0 \%)$ & $367(45.9 \%)$ & $121(45.5 \%)$ & $48(38.1 \%)$ & \\
\hline Race & & & & & 0.192 \\
\hline Black & $260(21.8 \%)$ & $160(19.9 \%)$ & $72(27.1 \%)$ & $28(22.2 \%)$ & \\
\hline White & $881(73.9 \%)$ & $606(75.8 \%)$ & $182(68.4 \%)$ & $93(73.8 \%)$ & \\
\hline Other & $51(4.3 \%)$ & $34(4.3 \%)$ & $12(4.5 \%)$ & $5(4.0 \%)$ & \\
\hline Tumor site & & & & & 0.228 \\
\hline Axial & $302(25.3 \%)$ & $207(25.9 \%)$ & $58(21.8 \%)$ & $37(29.4 \%)$ & \\
\hline Extremity & $890(74.7 \%)$ & $593(74.1 \%)$ & $208(78.2 \%)$ & $89(70.6 \%)$ & \\
\hline Surgical stage & & & & & 0.757 \\
\hline Regional & $514(43.1 \%)$ & $337(42.1 \%)$ & $116(43.6 \%)$ & $61(48.4 \%)$ & \\
\hline Localized & $400(33.6 \%)$ & $275(34.4 \%)$ & $87(32.7 \%)$ & $38(30.2 \%)$ & \\
\hline Distant & $218(23.3 \%)$ & $188(23.5 \%)$ & $63(23.7 \%)$ & $27(21.4 \%)$ & \\
\hline Surgery & & & & & 0.884 \\
\hline NO & $137(11.5 \%)$ & $92(11.5 \%)$ & $32(12.0 \%)$ & $13(10.3 \%)$ & \\
\hline YES & $1055(88.5 \%)$ & $708(88.5 \%)$ & $234(88.0 \%)$ & $113(89.7 \%)$ & \\
\hline Grade & & & & & 0.456 \\
\hline Well differentiated & $63(5.3 \%)$ & $41(5.1 \%)$ & $13(4.9 \%)$ & $9(7.1 \%)$ & \\
\hline Moderately differentiated & $85(7.1 \%)$ & $60(7.5 \%)$ & $17(6.4 \%)$ & $8(6.3 \%)$ & \\
\hline Poorly differentiated & $396(33.2 \%)$ & $261(32.6 \%)$ & $84(31.6 \%)$ & $51(40.5 \%)$ & \\
\hline Undifferentiated/anaplastic & $648(54.4 \%)$ & $438(54.8 \%)$ & $152(57.1 \%)$ & $58(46.0 \%)$ & \\
\hline
\end{tabular}


Table 1. Continued ...

\begin{tabular}{|c|c|c|c|c|c|}
\hline Variables & $\begin{array}{l}\text { Total cohort } \\
\mathrm{n}=1192 \\
\text { No. }(\%)\end{array}$ & $\begin{array}{c}\text { Development cohort }^{\mathrm{a}} \\
\mathbf{n}=\mathbf{8 0 0} \\
\text { No. }(\%)\end{array}$ & $\begin{array}{c}\text { Validation } \\
\text { cohort one }^{\text {a }} \\
n=266 \\
\text { No. }(\%)\end{array}$ & $\begin{array}{c}\text { Validation } \\
\text { cohort two }^{b} \\
n=126 \\
\text { No. }(\%)\end{array}$ & p-value \\
\hline Histology & & & & & 0.112 \\
\hline Conventional osteosarcoma & $783(65.7 \%)$ & $538(67.3 \%)$ & $161(60.5 \%)$ & $84(66.7 \%)$ & \\
\hline Chondroblastic osteosarcoma & $185(15.5 \%)$ & $130(16.3 \%)$ & $36(13.5 \%)$ & $19(15.1 \%)$ & \\
\hline Fibroblastic osteosarcoma & $36(3.0 \%)$ & $24(3.0 \%)$ & $10(3.8 \%)$ & $2(1.6 \%)$ & \\
\hline Telangiectatic osteosarcoma & $29(2.4 \%)$ & $18(2.3 \%)$ & $9(3.4 \%)$ & $2(1.6 \%)$ & \\
\hline Osteosarcoma in Paget disease of bone & $3(0.3 \%)$ & $3(0.4 \%)$ & $0(0 \%)$ & $0(\%)$ & \\
\hline Small cell osteosarcoma & $12(1.0 \%)$ & $6(0.8 \%)$ & $5(1.9 \%)$ & $1(0.8 \%)$ & \\
\hline Central osteosarcoma & $60(5.0 \%)$ & $30(3.8 \%)$ & $20(7.5 \%)$ & $10(7.9 \%)$ & \\
\hline Intraosseous well differentiated osteosarcoma & $2(0.2 \%)$ & $0(0 \%)$ & $2(0.8 \%)$ & $0(0 \%)$ & \\
\hline Parosteal osteosarcoma & $63(5.3 \%)$ & $40(5.0 \%)$ & $17(6.4 \%)$ & $6(4.8 \%)$ & \\
\hline Periosteal osteosarcoma & $12(1.0 \%)$ & $6(0.8 \%)$ & $5(1.9 \%)$ & $1(0.8 \%)$ & \\
\hline High grade surface osteosarcoma & $7(0.6 \%)$ & $5(0.6 \%)$ & $1(0.4 \%)$ & $1(0.8 \%)$ & \\
\hline AJCC stage & & & & & 0.788 \\
\hline $\mathrm{I}+\mathrm{II}$ & $930(78.0 \%)$ & $624(78.0 \%)$ & $205(77.1 \%)$ & $101(80.2 \%)$ & \\
\hline III+IV & $262(22.0 \%)$ & $176(22.0 \%)$ & $61(22.9 \%)$ & 25 (19.8\%) & \\
\hline TNM_T & & & & & 0.232 \\
\hline $\mathrm{T} 1$ & $481(40.4 \%)$ & $320(40.0 \%)$ & $102(38.3 \%)$ & $59(46.8 \%)$ & \\
\hline $\mathrm{T} 2$ & $639(53.6 \%)$ & $434(54.3 \%)$ & $141(53.0 \%)$ & $64(50.8 \%)$ & \\
\hline $\mathrm{T} 3$ & $31(2.6 \%)$ & $19(2.4 \%)$ & $10(3.8 \%)$ & $2(1.6 \%)$ & \\
\hline $\mathrm{Tx}$ & $41(3.4 \%)$ & $27(3.4 \%)$ & $13(4.9 \%)$ & $1(0.8 \%)$ & \\
\hline TNM_N & & & & & 0.113 \\
\hline N0 & $1161(97.4 \%)$ & 775 (96.9\%) & $260(97.7 \%)$ & $126(100.0 \%)$ & \\
\hline N1 & $31(2.6 \%)$ & $25(3.1 \%)$ & $6(2.3 \%)$ & $0(0 \%)$ & \\
\hline TNM_M & & & & & 0.909 \\
\hline M0 & $957(80.3 \%)$ & $641(80.1 \%)$ & $213(80.1 \%)$ & $103(81.7 \%)$ & \\
\hline M1 & $235(19.7 \%)$ & $159(19.9 \%)$ & 53 (19.9\%) & $23(18.3 \%)$ & \\
\hline Tumor size & & & & & 0.963 \\
\hline$\leq 9.6$ & $659(55.3 \%)$ & $437(54.6 \%)$ & $149(56.0 \%)$ & $71(56.3 \%)$ & \\
\hline $9.6-20.5$ & $430(36.1 \%)$ & $294(36.8 \%)$ & $93(35.0 \%)$ & $43(34.1 \%)$ & \\
\hline$\geq 20.5$ & $105(8.6 \%)$ & $69(8.6 \%)$ & $24(9.0 \%)$ & $12(9.6 \%)$ & \\
\hline
\end{tabular}

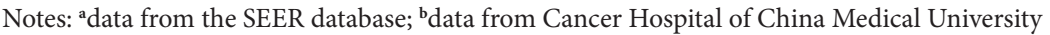

tumor site, and tumor size were confirmed as independent prognostic factors for OS (Table 2).

Similar statistical procedures were conducted to identify the independent prognostic factors for CSS. In univariate analyses, variables of age, gender, tumor site, surgical stage, surgery, grade, histology, AJCC stage, node involvement, metastasis, tumor size were also significantly associated with CSS (all $\mathrm{p}<0.05$ ). Among them, the variables of age, surgical stage, surgery, grade, tumor site, and tumor size were further identified as independent prognostic factors for CSS (Table 3 ).

Establishment and validation of nomograms for OS and CSS. According to the results from multivariate Cox proportional hazards analyses, age, surgical stage, surgery, grade, tumor site, and tumor size were finally incorporated to develop the prognostic nomograms for predicting the 3and 5-year OS and CSS in osteosarcoma patients (Figure 3). The detailed points of each selected variable were calculated and presented in Table 4 .
The internal and external validations of the developed predictive prognostic nomograms were conducted based on the data from the SEER database and our hospital. Internal validation in the development cohort revealed that the C-index values for the nomograms of OS and CSS were 0.818 (95\% CI 0.804-0.932) and 0.829 (95\% CI 0.814-0.844), respectively. Comparatively, the calculated C-index values for OS and CSS in the external validation cohort one was 0.843 (95\% CI 0.822-0.864) and 0.834 (95\% CI 0.807-0.861), respectively. While in external validation cohort two, the corresponding C-index values for OS and CSS were 0.736 (95\% CI $0.685-0.787$ ) and 0.782 (95\% CI $0.719-0.845$ ), respectively. The $\mathrm{C}$-index values from the three validation cohorts were all over 0.7 , indicating the favorable accuracy of our prognostic nomograms. Furthermore, the calibration plots also showed excellent agreement between the nomogram estimated survival and actual survival in 3- and 5-year OS and CSS (Figures 4A-4L). 
Table 2. Univariate and multivariate analyses of overall survival in the Development cohort.

\begin{tabular}{|c|c|c|c|}
\hline \multirow{2}{*}{ Variables } & \multirow{2}{*}{$\begin{array}{c}\text { Univariate analysis } \\
\text { p-value }\end{array}$} & \multirow{2}{*}{$\begin{array}{c}\text { Multivariate analysis } \\
\text { HR }(95 \% \mathrm{CI})\end{array}$} & \multirow{2}{*}{ p-value } \\
\hline & & & \\
\hline Age & $<0.001$ & & \\
\hline$\leq 26$ & & Reference & \\
\hline $26-61$ & & $2.046(1.423-2.940)$ & $<0.001$ \\
\hline$\geq 61$ & & $4.620(3.313-6.440)$ & $<0.001$ \\
\hline Gender & 0.001 & & \\
\hline Male & & Reference & \\
\hline Female & & $0.814(0.621-1.069)$ & 0.139 \\
\hline Race & 0.800 & & \\
\hline Black & & Reference & \\
\hline White & & $1.309(0.898-1.907)$ & 0.161 \\
\hline Other & & $1.003(0.572-1.755)$ & 0.992 \\
\hline Tumor site & $<0.001$ & & \\
\hline Axial & & Reference & \\
\hline Extremity & & $0.582(0.567-1.077)$ & $<0.001$ \\
\hline Surgical stage & $<0.001$ & & \\
\hline Regional & & Reference & \\
\hline Localized & & $0.871(0.589-1.287)$ & 0.487 \\
\hline Distant & & $2.442(1.263-4.721)$ & 0.007 \\
\hline Surgery & $<0.001$ & & \\
\hline NO & & Reference & \\
\hline YES & & $0.404(0.288-0.565)$ & $<0.001$ \\
\hline Grade & $<0.001$ & & \\
\hline Well differentiated & & Reference & \\
\hline Moderately differentiated & & $1.977(0.482-8.108)$ & 0.343 \\
\hline Poorly differentiated & & $5.909(1.754-19.897)$ & 0.004 \\
\hline Undifferentiated/anaplastic & & $5.035(1.512-16.768)$ & 0.008 \\
\hline Histology & $<0.001$ & & \\
\hline Conventional osteosarcoma & & Reference & \\
\hline Chondroblastic osteosarcoma & & $0.847(0.575-1.247)$ & 0.400 \\
\hline Fibroblastic osteosarcoma & & $0.909(0.437-1.890)$ & 0.799 \\
\hline Telangiectatic osteosarcoma & & $0.895(0.280-2.881)$ & 0.857 \\
\hline Osteosarcoma in Paget disease of bone & & $1.266(0.285-5.609)$ & 0.756 \\
\hline Small cell osteosarcoma & & $0.654(0.199-2.148)$ & 0.484 \\
\hline Central osteosarcoma & & $0.551(0.262-1.156)$ & 0.114 \\
\hline Intraosseous well differentiated osteosarcoma & & $0.869(0.000-4.56 \mathrm{E}+78)$ & 0.997 \\
\hline Parosteal osteosarcoma & & $0.854(0.237-3.070)$ & 0.808 \\
\hline Periosteal osteosarcoma & & $0.329(0.000-2.31 \mathrm{E}+56)$ & 0.991 \\
\hline High grade surface osteosarcoma & & $1.202(0.165-8.727)$ & 0.855 \\
\hline AJCC stage & $<0.001$ & & \\
\hline $\mathrm{I}+\mathrm{II}$ & & Reference & \\
\hline III+IV & & $3.652(0.651-8.192)$ & $<0.001$ \\
\hline TNM_T & 0.004 & & \\
\hline T1 & & Reference & \\
\hline $\mathrm{T} 2$ & & $0.828(0.498-1.375)$ & 0.466 \\
\hline T3 & & $0.997(0.427-2.325)$ & 0.994 \\
\hline $\mathrm{Tx}$ & & $0.954(0.472-1.930)$ & 0.897 \\
\hline TNM_N & 0.04 & & \\
\hline N0 & & Reference & \\
\hline N1 & & $0.916(0.483-1.736)$ & 0.789 \\
\hline TNM_M & $<0.001$ & & \\
\hline M0 & & Reference & \\
\hline M1 & & $1.024(0.417-2.506)$ & 0.959 \\
\hline Tumor size & $<0.001$ & & \\
\hline$\leq 9.6$ & & Reference & \\
\hline $9.6-20.5$ & & $1.658(0.996-2.756)$ & 0.051 \\
\hline$\geq 21.4$ & & $2.143(1.287-3.566)$ & 0.003 \\
\hline
\end{tabular}

Abbreviations: CI-confidence interval; HR-hazard ratio 
Table 3. Univariate and multivariate analyses of cancer-specific survival in the Development cohort.

\begin{tabular}{|c|c|c|c|}
\hline \multirow{2}{*}{ Variables } & \multirow{2}{*}{$\begin{array}{c}\text { Univariate analysis } \\
\text { p-value }\end{array}$} & \multirow{2}{*}{$\begin{array}{c}\text { Multivariate analysis } \\
\text { HR }(95 \% \mathrm{CI})\end{array}$} & \multirow{2}{*}{ p-value } \\
\hline & & & \\
\hline Age & $<0.001$ & & \\
\hline$\leq 26$ & & Reference & \\
\hline $26-61$ & & $2.039(1.361-3.055)$ & $<0.001$ \\
\hline$\geq 61$ & & $3.144(2.097-4.714)$ & $<0.001$ \\
\hline Gender & $<0.001$ & & \\
\hline Male & & Reference & \\
\hline Female & & $0.743(0.539-1.024)$ & 0.070 \\
\hline Race & 0.600 & & \\
\hline Black & & Reference & \\
\hline White & & $1.506(0.966-2.346)$ & 0.070 \\
\hline Other & & $1.099(0.570-2.120)$ & 0.777 \\
\hline Tumor site & $<0.001$ & & \\
\hline Axial & & Reference & \\
\hline Extremity & & $0.514(0.615-1.358)$ & $<0.001$ \\
\hline Surgical stage & $<0.001$ & & \\
\hline Regional & & Reference & \\
\hline Localized & & $1.041(0.648-1.672)$ & 0.867 \\
\hline Distant & & $2.720(1.179-6.269)$ & 0.018 \\
\hline Surgery & $<0.001$ & & \\
\hline NO & & Reference & \\
\hline YES & & $0.337(0.226-0.501)$ & $<0.001$ \\
\hline Grade & $<0.001$ & & \\
\hline Well differentiated & & Reference & \\
\hline Moderately differentiated & & $2.207(0.589-12.235)$ & $<0.001$ \\
\hline Poorly differentiated & & $5.307(2.573-9.881)$ & $<0.001$ \\
\hline Undifferentiated/anaplastic & & $4.435(2.014-7.495)$ & $<0.001$ \\
\hline Histology & $<0.001$ & & \\
\hline Conventional osteosarcoma & & Reference & \\
\hline Chondroblastic osteosarcoma & & $0.739(0.466-1.170)$ & 0.197 \\
\hline Fibroblastic osteosarcoma & & $0.693(0.277-1.733)$ & 0.433 \\
\hline Telangiectatic osteosarcoma & & $1.117(0.341-3.651)$ & 0.854 \\
\hline Osteosarcoma in Paget disease of bone & & $1.313(0.167-10.324)$ & 0.795 \\
\hline Small cell osteosarcoma & & $0.827(0.246-2.774)$ & 0.757 \\
\hline Central osteosarcoma & & $0.525(0.224-1.229)$ & 0.137 \\
\hline Intraosseous well differentiated osteosarcoma & & $0.197(0.000-3.895 \mathrm{E}+78)$ & 0.909 \\
\hline Parosteal osteosarcoma & & $0.773(0.160-3.737)$ & 0.749 \\
\hline Periosteal osteosarcoma & & $0.199(0.027-1.658)$ & 0.142 \\
\hline High grade surface osteosarcoma & & $1.016(0.032-6.326)$ & 0.938 \\
\hline AJCC stage & $<0.001$ & & \\
\hline $\mathrm{I}+\mathrm{II}$ & & Reference & \\
\hline III+IV & & $3.716(0.578-7.090)$ & $<0.001$ \\
\hline TNM_T & 0.001 & & \\
\hline $\mathrm{T} 1$ & & Reference & \\
\hline $\mathrm{T} 2$ & & $1.184(0.665-2.105)$ & 0.566 \\
\hline $\mathrm{T} 3$ & & $1.399(0.570-3.429)$ & 0.463 \\
\hline $\mathrm{Tx}$ & & $1.033(0.462-2.309)$ & 0.936 \\
\hline TNM_N & 0.09 & & \\
\hline N0 & & Reference & \\
\hline N1 & & $0.876(0.414-1.852)$ & 0.730 \\
\hline TNM_M & $<0.001$ & & \\
\hline M0 & & Reference & \\
\hline M1 & & $1.214(0.458-3.211)$ & 0.696 \\
\hline Tumor size & $<0.001$ & & \\
\hline$\leq 9.6$ & & Reference & \\
\hline $9.6-20.5$ & & $1.826(0.883-4.636)$ & 0.003 \\
\hline$\geq 21.4$ & & $3.942(1.122-6.359)$ & 0.017 \\
\hline
\end{tabular}

Abbreviations: CI-confidence interval; HR-hazard ratio 


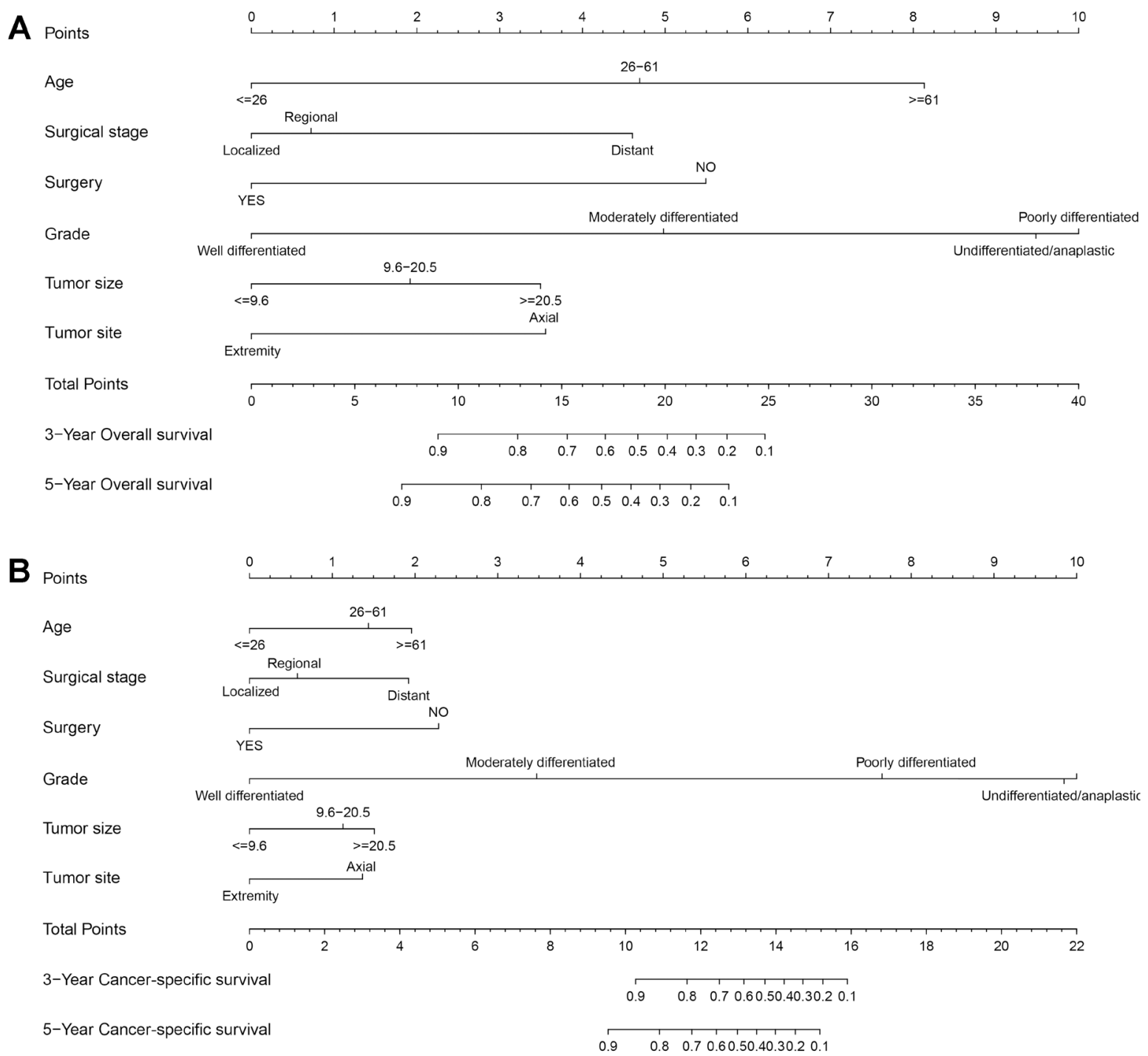

Figure 3. Nomograms to predict the overall survival (A) and cancer-specific survival (B) for patients with osteosarcoma. Firstly, by drawing a vertical line between each variable and the point scale, the points of this variable can be identified. Then, by projecting a vertical line from the total points scale, the sum of points of each variable included in the nomogram, to the OS and CSS scale, the individualized survival probability for an osteosarcoma patient can be obtained.

\section{Discussion}

Previous studies have suggested that multiple factors have prognostic or predictive values for the survival of osteosarcoma patients [41-43]. They can be roughly classified into seven groups based on their characteristics, as was mentioned in the introduction. Although these factors may form statistically significant associations with the survival of patients diagnosed with osteosarcoma, a single prognostic or predictive index may be inaccurate and unreliable in estimating an individual osteosarcoma patient's mediumor long-term survival. In addition, studies that incorporate these factors into a comprehensive and representative index or scoring system are scarce and thus impede its further application in individualized survival prediction. Fortunately, a nomogram is such a common statistical tool that can effectively integrate these prognostic factors together and provide satisfactory reliability, accuracy, and robustness in predicting an individual osteosarcoma patient's survival probability [44]. Kim et al. constructed and internally validated a prognostic nomogram that could predict the 5-year probability of metastasis for AJCC stage II extremity osteosarcoma patients $(\mathrm{n}=141)$ and this nomogram showed obvious superiority in metastasis risk estimation compared with the AJCC staging system or tumor necrosis rate [45]. In 2014, the author published another nomogram to evaluate 
Table 4. Points of each variable included in the nomograms.

\begin{tabular}{|c|c|c|}
\hline Variables & $\begin{array}{c}\text { Overall } \\
\text { survival } \\
\text { nomogram }\end{array}$ & $\begin{array}{c}\text { Cancer-specific } \\
\text { survival } \\
\text { nomogram } \\
\end{array}$ \\
\hline \multicolumn{3}{|l|}{ Age (years) } \\
\hline$\leq 26$ & 0 & 0 \\
\hline $26-61$ & 4.5 & 1.5 \\
\hline$\geq 61$ & 8.0 & 2.0 \\
\hline \multicolumn{3}{|l|}{ Surgical stage } \\
\hline Regional & 1.0 & 0.5 \\
\hline Localized & 0 & 0 \\
\hline Distant & 4.5 & 2.0 \\
\hline \multicolumn{3}{|l|}{ Surgery } \\
\hline NO & 5.5 & 2.0 \\
\hline YES & 0 & 0 \\
\hline \multicolumn{3}{|l|}{ Grade } \\
\hline Well differentiated & 0 & 0 \\
\hline Moderately differentiated & 5.0 & 3.5 \\
\hline Poorly differentiated & 9.5 & 7.5 \\
\hline Undifferentiated/anaplastic & 10.0 & 10.0 \\
\hline \multicolumn{3}{|l|}{ Tumor size $(\mathrm{cm})$} \\
\hline$\leq 9.6$ & 0 & 0 \\
\hline $9.6-20.5$ & 2.0 & 1.0 \\
\hline$\geq 21.4$ & 3.5 & 1.5 \\
\hline \multicolumn{3}{|l|}{ Tumor site } \\
\hline Extremity & 0 & 0 \\
\hline Axial & 3.5 & 1.5 \\
\hline
\end{tabular}

the risk of metastasis in patients diagnosed with Enneking stage IIB extremity osteosarcoma ( $\mathrm{n}=91)$ who received limb salvage surgery [46]. However, the two studies just focused on AJCC stage II or Enneking stage IIB extremity osteosarcoma patients, so the representativeness of the patients was limited. Moreover, several other limitations also existed in these studies, including without external validation based on other populations and relatively small sample sizes. Therefore, their results might not apply to other populations due to potential bias. Xia et al. also developed a nomogram that included an inflammatory response marker of neutrophil-to-lymphocyte ratio (NLR) to predict the survival of osteosarcoma patients who underwent curative surgery [9]. However, this study devised a nomogram with only three prognostic factors included, namely NLR, tumor stage, and initial metastases, and thus crippled its clinical and practical values for its inability to represent the overall condition of an osteosarcoma patient. Therefore, the above-mentioned nomograms should be validated in larger and different populations before its application in clinical practice. So, in our study, by integrating pivotal prognostic and determinant variables together, we first devel-
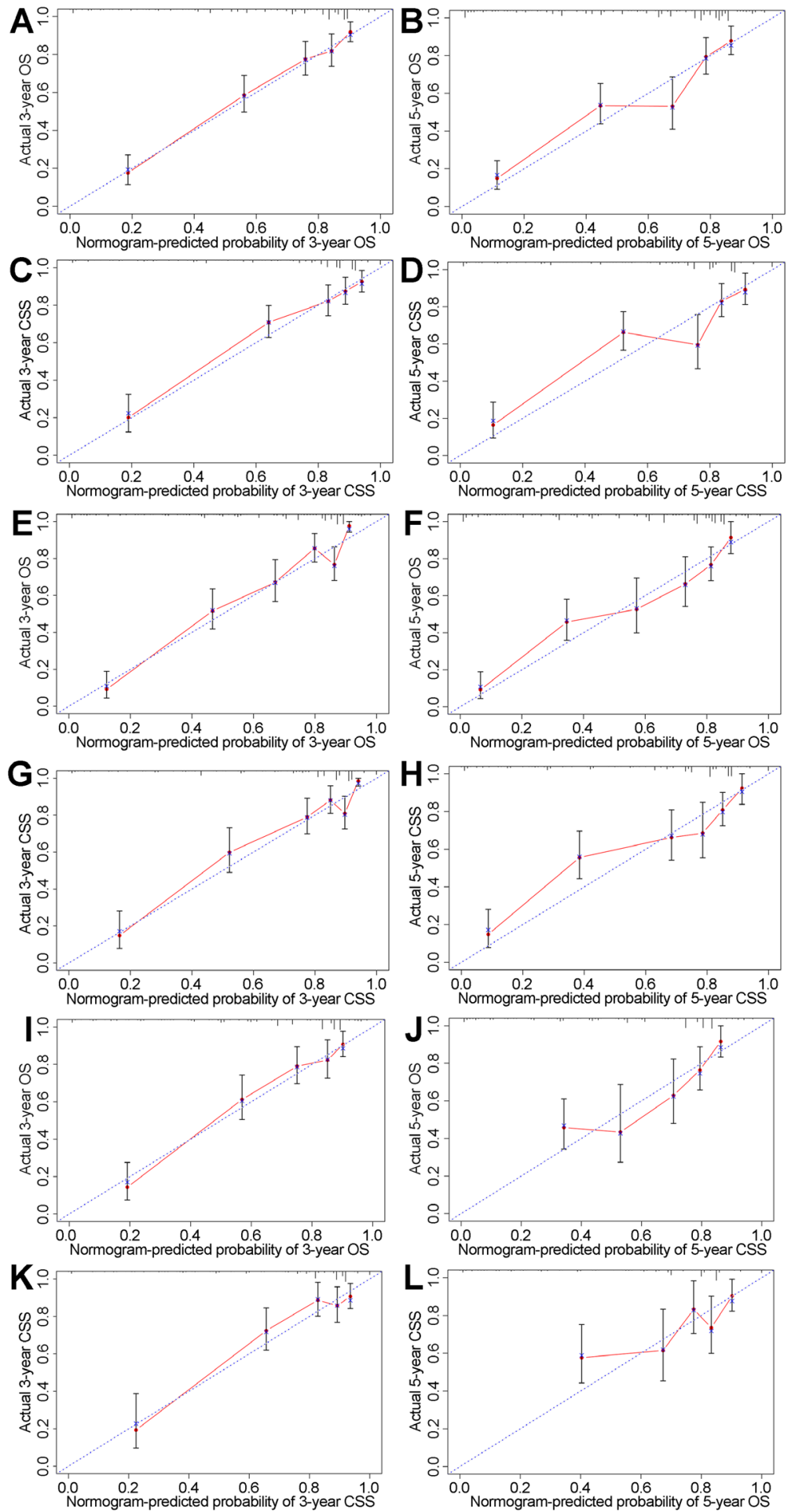

Figure 4. The twelve graphs show the calibration plots of internal validation and external validation for the 3- and 5-year overall survival $(O S)$ and cancer-specific survival (CSS). A-D) The first four calibration curves present the internal validation for 3- and 5-year OS and CSS based on the data from the SEER database. E-H) The middle four calibration curves show the external validation for 3- and 5-year OS and CSS based on the data from the SEER database. I-L) The last four calibration curves also reveal the external validation, data come from our hospital, for 3-and 5-year OS and CSS. 
oped and internally validated the prognostic nomograms to evaluate the 3- and 5-year OS and CSS rates for patients with osteosarcoma based on the data of a cohort from the SEER database. Then, we externally validated the established nomograms with the data of another cohort from the SEER database. Finally, we performed further external validation of the nomograms with the data from our hospital.

Based on results from univariate and multivariate Cox analyses, several clinicopathological factors were finally identified to be independently associated with the survival of patients with osteosarcoma, including patient age, surgical stage, surgery, tumor grade, tumor size, tumor site, and AJCC stage. In the present study, increasing age was significantly associated with worse survival status in osteosarcoma patients. Similar trends have been reported in previous studies, but the cut-off values for patient age may vary in different studies. Zheng et al. reported that patients with osteosarcoma older than 51 had worse survival time and Song et al. proposed that patients' age over 40 years old was an independent risk factor for poor survival $[39,40]$. Similarly, another study based on the SEER database analyses and performed by Fu et al. also suggested that patients older than 25 years almost suffered 2-3 times increased risks to get poorer survival [47]. In our study, we identified the age of 26 and 61 as the best cut-off points for risk stratification by using the X-tile software and significant differences in OS and CSS were found between patients $\leq 26$ years, $26-61$ years, and $\geq 61$ years. The distinction in cut-off values of age at disease onset can be explained to some extent that it was determined by the sample size and study populations in different studies. The surgical stage, or the extent of disease, was also an independent risk factor for worse survival outcomes. Patients who suffered metastatic lesions or accompanied by regional lymph nodes involvement usually had a poorer prognosis than those with a localized disease or without lymph nodes metastases [6, 39, 40, 47, 48]. Consistent with previous findings, our study also revealed that osteosarcoma patients with regional or distant disease had higher mortality than patients with localized disease.

Currently, neoadjuvant chemotherapy followed by surgical resection remains the standard modality for patients with non-metastatic osteosarcoma [4]. Our study also validated and confirmed the beneficial effects of surgery on the survival of patients with osteosarcoma who received treatment of chemotherapy based on the results of multivariate analyses. In our study, we also identified tumor grade as independent prognostic factors for the survival outcomes of osteosarcoma patients, which was in line with the results in previous studies. Patients with a poorly differentiated or undifferentiated histology grade were more prone to get worse OS and CSS than patients with well-differentiated or moderately differentiated histology grade. It was demonstrated by Wang et al. that high-grade osteosarcoma had significantly worse survival outcomes compared with low-grade osteosarcoma [49]. With respect to histology subtypes, the report from Jawad et al. demonstrated that Paget's osteosarcoma was significantly related to poorer prognosis compared with other histological subtypes and this result was further confirmed by the report of Damron et al. [50,51]. Our study also found that the periosteal osteosarcoma had a significantly better prognosis than all other histological subtypes of osteosarcoma, which was similar to the results reported by Zheng et al. [39].

In previous studies, axial tumor locations and large tumor sizes were deemed as the worst prognostic factors for the survival outcomes of patients with osteosarcoma [52-55]. Our study suggested that patients with axial tumors or larger tumors had decreased survival time compared with patients with extremity tumors or smaller tumors. Larger tumor sizes and axial tumor locations all impose several disadvantages for osteosarcoma patients, including increased risk for metastasis, soared difficulties for surgical resection, more difficulties to achieve adequate surgical margins, the rich blood supply to accelerate the process of elimination and metabolism of chemotherapeutic agents, and so on. Fujiwara et al. studied the association between proximity to the major vessels and the risk of local recurrence and survival in 226 patients with high-grade non-metastatic osteosarcoma [54]. Based on the examination of preoperative Magnetic Resonance Imaging (MRI), the vascular proximity was categorized into four types: type 1 with proximity $>5 \mathrm{~mm}$; type 2 with proximity $\leq 5 \mathrm{~mm},>0 \mathrm{~mm}$; type 3 with tumors attached to major vessels; type 4 with tumors surrounded major vessels. The results revealed that the limb salvage rate and the 5-year OS rate were larger in patients with longer tumor-to-vessel proximities than those with shorter proximities $(92 \%, 88 \%, 51 \%, 0 \%$ vs. $82 \%, 77 \%, 57 \%, 67 \%)$. Moreover, the local recurrence rate was also higher in patients with shorter tumor-to-vessel proximities (types 1 to 3; 7\%, 8\%, and 22\%). Furthermore, Bielack et al. and Duchman et al. also demonstrated that patients with larger tumors adjacent to trunk bones are more likely to develop metastases during chemotherapy $[52,56]$. Besides, our study also found that the AJCC stage was also independently related to the prognosis of osteosarcoma patients. Patients with a higher AJCC stage were usually accompanied by a poorer prognosis and survival. In the present study, the AJCC stage was not included in the nomograms for it was a comprehensive evaluating system based on tumor size, lymph node involvement, and metastasis. If it was included for the construction of the nomogram, the weight coefficient for tumor size and surgical stage may alter for the reasons for repeated calculation of weight coefficient for them. The established nomograms for osteosarcoma patients in previous studies also did not incorporate the AJCC stage into nomograms $[39,40]$.

Different from previous studies, we performed external validation of the established nomograms with the data of a different cohort from our hospital. Previously, we have conducted a study to explore the predictive values of pre-treatment Naples prognostic score (NPS) in patients with osteosarcoma in a cohort of 133 patients [57]. We constructed two prediction models based on the multivariate Cox 
analyses, namely the conventional prediction model (model A) based on prognostic factors of Enneking stage, metastasis, local recurrence, and NLR, and the combined prediction model (model B) based on prognosticators of Enneking stage, metastasis, local recurrence, NLR, and NPS. The results from our previous study demonstrated that NPS based nomograms got better time-dependent AUCs than conventional nomograms only established by clinical characteristics. The AUCs for model A were $0.802-0.878$ and $0.773-0.840$ for OS and PFS, while the AUCs for model B were 0.6640.713 and $0.773-0.718$ for OS and PFS, respectively. In this study, the C-indexes of the nomograms in the development set ( 0.818 for OS, 0.829 for CSS) and the two validation sets (validation set one, 0.843 for OS and 0.834 for CSS; validation set two, 0.736 for OS and 0.782 for CSS) were all over 0.70 . By contrast, the predictive performances of the nomograms in this study and our previous study were both reliable and effective. However, the nomograms based on a large cohort from the SEER database seemed to be slightly better than the nomograms based on the small cohort from our hospital. This is within our anticipation because prediction models based on a larger cohort are more prone to harvest better prediction performances than those established from a relatively smaller cohort. Anyhow, the indexes (AUC or C-index) representing the predictive accuracy in our current study and previous study were both larger than 0.7 , which indicates these nomograms were all reliable and effective.

Although the established nomograms in our study showed good performance in predicting the OS and CSS for osteosarcoma patients, several limitations should also be taken into consideration. First, the detailed data on chemotherapy and radiotherapy were incomplete and limited in the SEER database, leading to the loss of some important variables for nomogram construction and possibilities to cause some relevant bias. Second, some pivotal serological biomarkers were not included in our nomogram, such as lactate dehydrogenase (LDH) and alkaline phosphatase (ALP), for such variables were not available and cannot be extracted from the SEER database. Finally, other factors, like local recurrence and multiple metastases, were also important endpoints for osteosarcoma patients, but they were also unextractable in the SEER database, which impeded the process for further analysis. Despite these limitations, the nomograms also showed good predictive performance in external validation with the data from our hospital $(\mathrm{C}$-index $=0.736)$.

In summary, we firstly established prognostic nomograms to predict the 3- and 5-year OS and CSS rates for patients with osteosarcoma based on the data from the SEER database. Then, the constructed nomograms were internally and externally validated with data from the SEER database and our hospital, which all showed good predictive performance for osteosarcoma patients. With this predictive tool, the 3-and 5 -year survival of an individual osteosarcoma patient can be estimated, enabling oncologists to evaluate individualized survival probability and identify mortality risk stratification.

\section{References}

[1] MIRABELLO L, TROISI RJ, SAVAGE SA. Osteosarcoma incidence and survival rates from 1973 to 2004: data from the Surveillance, Epidemiology, and End Results Program. Cancer-Am Cancer Soc 2009; 115: 1531-1543. https://doi. org/10.1002/cncr.24121

[2] MIRABELLO L, TROISI RJ, SAVAGE SA. International osteosarcoma incidence patterns in children and adolescents, middle ages and elderly persons. Int J Cancer 2009; 125: 229-234. https://doi.org/10.1002/ijc.24320

[3] DAHLIN DC, UNNI KK. Osteosarcoma of bone and its important recognizable varieties. Am J Surg Pathol 1977; 1: 61-72. https://doi.org/10.1097/00000478-197701010-00007

[4] ISAKOFF MS, BIELACK SS, MELTZER P, GORLICK R. Osteosarcoma: Current Treatment and a Collaborative Pathway to Success. J Clin Oncol 2015; 33: 3029-3035. https://doi. org/10.1200/JCO.2014.59.4895

[5] BACCI G, FERRARI S, BERTONI F, RUGGIERI P, PICCI P et al. Long-term outcome for patients with nonmetastatic osteosarcoma of the extremity treated at the istituto ortopedico rizzoli according to the istituto ortopedico rizzoli/osteosarcoma-2 protocol: an updated report. J Clin Oncol 2000; 18: 4016-4027. https://doi.org/10.1200/JCO.2000.18.24.4016

[6] MIALOU V, PHILIP T, KALIFA C, PEROL D, GENTET JC et al. Metastatic osteosarcoma at diagnosis: prognostic factors and long-term outcome--the French pediatric experience. Cancer-Am Cancer Soc 2005; 104: 1100-1109. https:// doi.org/10.1002/cncr.21263

[7] KAGER L, ZOUBEK A, POTSCHGER U, KASTNER U, FLEGE $S$ et al. Primary metastatic osteosarcoma: presentation and outcome of patients treated on neoadjuvant Cooperative Osteosarcoma Study Group protocols. J Clin Oncol 2003; 21: 2011-2018. https://doi.org/10.1200/JCO.2003.08.132

[8] LIX,TIANF, WANGF,LIY.SerumC-reactive protein and overall survival of patients with osteosarcoma. Tumour Biol 2015; 36: 5663-5666. https://doi.org/10.1007/s13277-015-3240-6

[9] XIA WK, LIU ZL, SHEN D, LIN QF, SU J et al. Prognostic performance of pre-treatment NLR and PLR in patients suffering from osteosarcoma. World J Surg Oncol 2016; 14: 127. https://doi.org/10.1186/s12957-016-0889-2

[10] LIU B, HUANG Y, SUN Y, ZHANG J, YAO Y et al. Prognostic value of inflammation-based scores in patients with osteosarcoma. Sci Rep 2016; 6: 39862. https://doi.org/10.1038/ srep39862

[11] TENARDI RD, FRUHWALD MC, JURGENS H, HERTROIJS D, BAUER J. Nutritional status of children and young adults with Ewing sarcoma or osteosarcoma at diagnosis and during multimodality therapy. Pediatr Blood Cancer 2013; 60: 166. https://doi.org/ 10.1002/pbc.24304

[12] SUN K, CHEN S, XU J, LI G, HE Y. The prognostic significance of the prognostic nutritional index in cancer: a systematic review and meta-analysis. J Cancer Res Clin Oncol 2014; 140: 1537-1549. https://doi.org/10.1016/j.ijsu.2019.05.018

[13] BOUILLANNE O, MORINEAU G, DUPONT C, COULOMBEL I, VINCENT JP et al. Geriatric Nutritional Risk Index: a new index for evaluating at-risk elderly medical patients. Am J Clin Nutr 2005; 82: 777-783. https://doi. org/10.1093/ajcn/82.4.777 
[14] LI Y, NAKKA M, KELLY AJ, LAU CC, KRAILO M et al. p27 Is a Candidate Prognostic Biomarker and Metastatic Promoter in Osteosarcoma. Cancer Res 2016; 76: 4002-4011. https://doi.org/10.1158/0008-5472.CAN-15-3189

[15] MA H, SEEBACHER NA, HORNICEK FJ, DUAN Z. Cyclindependent kinase 9 (CDK9) is a novel prognostic marker and therapeutic target in osteosarcoma. Ebiomedicine 2019; 39: 182-193. https://doi.org/10.1016/j.ebiom.2018.12.022

[16] KUBISTA B, EROVIC BM, KLINGER H, SULZBACHER I, TRIEB K. CD9 expression is not a prognostic factor in human osteosarcoma. Cancer Lett 2004; 209: 105-110. https:// doi.org/ 10.1016/j.canlet.2003.11.038

[17] ZHANG YL, PU YC, WANG J, LI ZC, WANG HL. Research progress regarding the role of long non-coding RNAs in osteosarcoma. Oncol Lett 2020; 20: 2606-2612. https://doi. org/10.3892/ol.2020.11807

[18] LIU W, ZHAO X, ZHANG YJ, FANG GW, XUE Y. MicroRNA-375 as a potential serum biomarker for the diagnosis, prognosis, and chemosensitivity prediction of osteosarcoma. J Int Med Res 2018; 46: 975-983. https://doi. org/10.1177/0300060517734114

[19] LI S, GAO Y, WANG Y, WANG K, DAI ZP et al. Serum microRNA-17 functions as a prognostic biomarker in osteosarcoma. Oncol Lett 2016; 12: 4905-4910. https://doi. org/10.3892/ol.2016.5362

[20] WU PF, DAI ZT, LIU WD, ZHAO ZX, KONG YH. Elevated long noncoding RNA HAGLROS expression correlates with clinical progression and prognosis in osteosarcoma. Eur Rev Med Pharmacol Sci 2019; 23: 1428-1433. https://doi. org/10.26355/eurrev_201902_17099

[21] KUN-PENG Z, CHUN-LIN Z, JIAN-PING H, LEI Z. A novel circulating hsa_circ_0081001 act as a potential biomarker for diagnosis and prognosis of osteosarcoma. Int J Biol Sci 2018; 14: 1513-1520. https://doi.org/10.7150/ijbs.27523

[22] BUDDINGH EP, KUIJJER ML, DUIM RA, BURGER H, AGELOPOULOS $\mathrm{K}$ et al. Tumor-infiltrating macrophages are associated with metastasis suppression in high-grade osteosarcoma: a rationale for treatment with macrophage activating agents. Clin Cancer Res 2011; 17: 2110-2119. https:// doi.org/10.1158/1078-0432.CCR-10-2047

[23] GOMEZ-BROUCHET A, ILLAC C, GILHODES J, BOUVIER C, AUBERT S et al. CD163-positive tumor-associated macrophages and CD8-positive cytotoxic lymphocytes are powerful diagnostic markers for the therapeutic stratification of osteosarcoma patients: An immunohistochemical analysis of the biopsies fromthe French OS2006 phase 3 trial. Oncoimmunology 2017; 6: e1331193. https://doi.org/10.108 0/2162402X.2017.1331193

[24] KOIRALA P, ROTH ME, GILL J, PIPERDI S, CHINAI JM et al. Immune infiltration and PD-L1 expression in the tumor microenvironment are prognostic in osteosarcoma. Sci Rep 2016; 6: 30093. https://doi.org/10.1038/srep30093

[25] DUMARS C, NGYUEN JM, GAULTIER A, LANEL R, CORRADINI N et al. Dysregulation of macrophage polarization is associated with the metastatic process in osteosarcoma. Oncotarget 2016; 7: 78343-78354. https://doi.org/10.18632/ oncotarget.13055
[26] CHUI MH, KANDEL RA, WONG M, GRIFFIN AM, BELL RS et al. Histopathologic Features of Prognostic Significance in High-Grade Osteosarcoma. Arch Pathol Lab Med 2016; 140: 1231-1242. https://doi.org/10.5858/arpa.2015-0389$\mathrm{OA}$

[27] BRAMER JA, ABUDU AA, GRIMER RJ, CARTER SR, TILLMAN RM. Do pathological fractures influence survival and local recurrence rate in bony sarcomas? Eur J Cancer 2007; 43: 1944-1951. https://doi.org/10.1016/j. ejca.2007.07.004

[28] SHIN KH, MOON SH, SUH JS, YANG WI. Tumor volume change as a predictor of chemotherapeutic response in osteosarcoma. Clin Orthop Relat Res 2000; 376: 200-208. https://doi.org/10.1097/00003086-200007000-00027

[29] SEKER MM, SEKER A, AKSOY S, OZDEMIR N, UNCU D et al. Clinicopathologic features and prognosis of osteosarcoma in Turkish adults. Asian Pac J Cancer Prev 2014; 15: 3537-3540. https://doi.org/10.7314/apjcp.2014.15.8.3537

[30] CATES JM, SCHOENECKER JG. Proximal location in extremity long bones is a poor prognostic factor for osteosarcoma: A retrospective cohort study of 153 patients. Acta Oncol 2016; 55: 1036-1039. https://doi.org/10.3109/028418 6X.2016.1156740

[31] OGURA K, FUJIWARA T, YASUNAGA H, MATSUI H, JEON DG et al. Development and external validation of nomograms predicting distant metastases and overall survival after neoadjuvant chemotherapy and surgery for patients with nonmetastatic osteosarcoma: A multi-institutional study. Cancer-Am Cancer Soc. 2015; 121: 3844-3852. https://doi.org/10.1002/cncr.29575

[32] BERTRAND TE, CRUZ A, BINITIE O, CHEONG D, LETSON GD et al. Do Surgical Margins Affect Local Recurrence and Survival in Extremity, Nonmetastatic, High-grade Osteosarcoma? Clin Orthop Relat Res 2016; 474: 677-683. https://doi.org/10.1007/s11999-015-4359-x

[33] GUO J, REDDICK WE, GLASS JO, JI Q, BILLUPS CA et al. Dynamic contrast-enhanced magnetic resonance imaging as a prognostic factor in predicting event-free and overall survival in pediatric patients with osteosarcoma. Cancer-Am Cancer Soc 2012; 118: 3776-3785. https://doi.org/10.1002/ cncr.26701

[34] IM HJ, ZHANG Y, WU H, WU J, DAW NC et al. Prognostic Value of Metabolic and Volumetric Parameters of FDG PET in Pediatric Osteosarcoma: A Hypothesis-generating Study. Radiology 2018; 287: 303-312. https://doi.org/10.1148/radiol.2017162758

[35] KUBO T, FURUTA T, JOHAN MP, OCHI M. Prognostic significance of (18)F-FDG PET at diagnosis in patients with soft tissue sarcoma and bone sarcoma; systematic review and meta-analysis. Eur J Cancer 2016; 58: 104-111. https://doi. org/10.1016/j.ejca.2016.02.007

[36] ZHONG J, ZHENG Q, AN T, ZHAO J, WU M et al. Nomogram to predict cause-specific mortality in extensive-stage small cell lung cancer: A competing risk analysis. Thorac Cancer 2019; 10: 1788-1797. https://doi.org/10.1111/17597714.13148 
[37] WANG J, LI Y, FU W, ZHANG Y, JIANG J et al. Prognostic nomogram based on immune scores for breast cancer patients. Cancer Med 2019; 8: 5214-5222. https://doi. org/10.1002/cam 4.2428

[38] CHEN QY, ZHONG Q, WANG W, DESIDERIO J, LIU ZY et al. Development and external validation of a nomogram for predicting the conditional probability of survival after D2 lymphadenectomy for gastric cancer: A multicentre study. Eur J Surg Oncol 2019; 45: 1934-1942. https://doi. org/10.1016/j.ejso.2019.04.003

[39] ZHENG W, HUANG Y, CHEN H, WANG N, XIAO W et al. Nomogram application to predict overall and cancer-specific survival in osteosarcoma. Cancer Manag Res 2018; 10: 54395450. https://doi.org/10.2147/CMAR.S177945

[40] SONG K, SONG J, CHEN F, LIN K, MA X et al. Prognostic nomograms for predicting overall and cancer-specific survival of high-grade osteosarcoma patients. J Bone Oncol 2018; 13: 106-113. https://doi.org/10.1016/j.jbo.2018.09.012

[41] ZAMBORSKY R, KOKAVEC M, HARSANYI S, DANISOVIC L. Identification of Prognostic and Predictive Osteosarcoma Biomarkers. Med Sci (Basel) 2019; 72: 28. https://doi. org/10.3390/medsci7020028

[42] TAN G, GERRAND CH, RANKIN KS. Blood-borne biomarkers of osteosarcoma: A systematic review. Pediatr Blood Cancer 2019; 66: e27462. https://doi.org/10.1002/pbc.27462

[43] BAKHSHI S, RADHAKRISHNAN V. Prognostic markers in osteosarcoma. Expert Rev Anticancer Ther 2010; 10: 271287. https://doi.org/ 10.1586/era.09.186

[44] BALACHANDRAN VP, GONEN M, SMITH JJ, DEMATTEO RP. Nomograms in oncology: more than meets the eye. Lancet Oncol 2015; 16: e173-e180. https://doi.org/10.1016/ S1470-2045(14)71116-7

[45] KIM MS, LEE SY, LEE TR, CHO WH et al. Prognostic nomogram for predicting the 5 -year probability of developing metastasis after neo-adjuvant chemotherapy and definitive surgery for AJCC stage II extremity osteosarcoma. Ann Oncol 2009; 20: 955-960. https://doi.org/10.1093/annonc/ $\operatorname{mdn} 723$

[46] KIM SH, SHIN KH, KIM HY, CHO YJ, NOH JK et al. Postoperative nomogram to predict the probability of metastasis in Enneking stage IIB extremity osteosarcoma. Bmc Cancer. 2014; 14: 666. https://doi.org/10.1200/JCO.2000.18.24.4016

[47] FU P, SHI Y, CHEN G, FAN Y et al. Prognostic Factors in Patients With Osteosarcoma With the Surveillance, Epidemiology, and End Results Database. Technol Cancer Res T 2020; 19: 1079215349. https://doi.org/10.1177/1533033820947701
[48] CLARK JC, DASS CR, CHOONG PF. A review of clinical and molecular prognostic factors in osteosarcoma. J Cancer Res Clin Oncol 2008; 134: 281-297. https://doi.org/10.1007/ s00432-007-0330-X

[49] WANG Z, LI S, LI Y, LIN N, HUANG X et al. Prognostic factors for survival among patients with primary bone sarcomas of small bones. Cancer Manag Res 2018; 10: 1191-1199. https://doi.org/10.2147/CMAR.S163229

[50] JAWAD MU, CHEUNG MC, CLARKE J, KONIARIS LG, SCULLY SP. Osteosarcoma: improvement in survival limited to high-grade patients only. J Cancer Res Clin Oncol 2011; 137: 597-607. https://doi.org/10.1007/s00432-010-0923-7

[51] DAMRON TA, WARD WG, STEWART A. Osteosarcoma, chondrosarcoma, and Ewing's sarcoma: National Cancer Data Base Report. Clin Orthop Relat Res 2007; 459: 40-47. https://doi.org/10.1097/BLO.0b013e318059b8c9

[52] BIELACK SS, KEMPF-BIELACK B, DELLING G, EXNER GU, FLEGE S et al. Prognostic factors in high-grade osteosarcoma of the extremities or trunk: an analysis of 1,702 patients treated on neoadjuvant cooperative osteosarcoma study group protocols. J Clin Oncol 2002; 20: 776-790. https://doi.org/10.1200/JCO.2002.20.3.776

[53] BERNER K, BRULAND OS. Prognostic Impact of Proximal Versus Distal Localization in Extremity Long Bone Osteosarcomas. Anticancer Res 2019; 39: 2459-2466. https://doi. org/10.21873/anticanres.13365

[54] FUJIWARA T, MEDELLIN MR, SAMBRI A, TSUDA Y, BALKO J et al. Preoperative surgical risk stratification in osteosarcoma based on the proximity to the major vessels. Bone Joint J 2019; 101-B: 1024-1031. https://doi. org/10.1302/0301-620X.101B8.BJJ-2018-0963.R1

[55] JAFFE N, JAFFE DM. Tumor size and prognosis in aggressively treated osteosarcoma. J Clin Oncol 1996; 14: 23992400. https://doi.org/10.1200/JCO.1996.14.8.2399

[56] DUCHMAN KR, GAO Y, MILLER BJ. Prognostic factors for survival in patients with high-grade osteosarcoma using the Surveillance, Epidemiology, and End Results (SEER) Program database. Cancer Epidemiol 2015; 39: 93-599. https:// doi.org/10.1016/j.canep.2015.05.001

[57] YANG QK, CHEN T, YAO ZX, ZHANG XJ. Prognostic value of pre-treatment Naples prognostic score (NPS) in patients with osteosarcoma. World J Surg Oncol 2020; 18: 24. https:// doi.org/10.1186/s12957-020-1789-Z 